\title{
Size Effects on the Growth Mode and Roughness of Sub-Micron Structures Grown by Selective Area Epitaxy
}

\author{
H.R. Gutiérrez*, M.A. Cotta*, W.M. Nakaema*, M.M.G.de Carvalho*, and A.L. Gobbi** \\ *Instituto de Física "Gleb Wataghin" \\ UNICAMP, IFGW, DFA/LPD, CP 6165, 130831-970 Campinas, SP, Brazil \\ em ${ }^{* *} C P q D /$ Telebras - LNLS - Campinas, SP, Brazil
}

Received February 8, 1999

\begin{abstract}
We have observed changes in the morphology of InP films grown in epi-ready substrates and on patterns created by photo and plow-mode AFM lithography. These changes are attributed to different levels of contamination of the surface, which favor nucleation of clusters over a two-dimensional film for the lithography-processed surface. The area of nucleation is smaller than the dimensions of the patterns created by lithography, so no size effects due to the presence of the pattern could be observed. The film morphologies exhibited well defined exponents for system sizes smaller than 0.2 $\mu \mathrm{m}$ but no defined growth exponent.
\end{abstract}

\section{Introduction}

The study of roughening and formation of large-scale morphologies, during epitaxial growth, has received considerable attention in the last years. Many works, both theoretical and experimental, have investigated this problem with a variety of materials and growth conditions. In particular, the study of the scaling properties in these processes has become a useful instrument for the identification of the dominant growth mechanisms, by comparing with the roughness and growth exponents predicted for the different continuum and discrete models existing in the literature [1]. On the other hand, the fabrication of monolithically integrated electronic devices involves many steps in the process (photolithography, cleaning, etching, etc), which can modify the starting conditions for the epitaxial growths. Moreover, larger integration levels require a continuous reduction of the growing areas. These two factors may induce changes in the morphology of as-growing surfaces in relation with the samples obtained from the growth on larger areas and epi-ready substrates. In this work, the influence of the surface size and processing on the morphology of III-V compound films grown by Selective Area Epitaxy (SAE) technique is studied. SAE allows the growth on controlled areas of the substrate using openings in a dielectric mask. The as-deposited dielectric surface does not catalyze the cracking of metallorganic precursors used for growth in Chemical Vapor De- position and Chemical Beam Epitaxy (CBE) systems. The whole process required to provide the sub-micron openings in this dielectric mask involves photolithography to create pre-localization patterns, nanolithography by Atomic Force Microscope (AFM) in the plow mode, transfer of the nanopattern to the underlying dielectric film by corrosion and cleaning of residues left by these procedures. The morphologies and scaling behavior were studied by AFM. From the AFM data, surface roughness (1) and height-difference correlation function (2) were calculated as a function of the system size L. In equations (1) and (2), $\mathrm{h}$ is the film thickness, $\mathrm{x}$ the position at the surface and $\mathrm{l}=\left(\mathrm{x}-\mathrm{x}^{\prime}\right)[1]$.

$$
\begin{gathered}
W_{L}^{2}(l, t) \equiv<\left[h(x, t)-h_{l}(x, t)\right]^{2}>_{x} \\
C(l) \equiv\left[<\left(h(x)-h\left(x^{\prime}\right)\right)^{2}>_{x}\right]^{1 / 2}
\end{gathered}
$$

\section{Experimental}

The samples were grown on (001) InP substrates misoriented $2^{\circ}$ off towards [100](A-surface) using a CBE system. Trimethylindium (TMI) and thermally decomposed Phosphine were the group III and V sources, respectively. The substrate native oxides were removed by heating the substrate during 5 minutes at $535^{\circ} \mathrm{C}$ in the $\mathrm{CBE}$ chamber under $P_{2}$ overpressure. During the growth the substrate temperature was $490^{\circ} \mathrm{C}$ and the growth rate $3.8 \AA / \mathrm{sec}$. At these conditions a transition in growth mode (from two to three-dimensional) 
is observed with the resulting morphologies showing a rippled (periodical multi-terraces) structures elongated along the [011] direction[2].

Before the growth, some samples were processed by conventional photolithography in order to obtain the pre-localization patterns. The AFM lithography process was used to create patterns of different sizes ranging from $2 \mu \mathrm{m}$ to $0.5 \mu \mathrm{m}$. The as-processed samples were cleaned with Trichloroethylene, acetone, methanol and finally with a low power $\mathrm{O}_{2}$ plasma during $20 \mathrm{~min}-$ utes. Using the above growth conditions, samples deposited on both epi-ready and processed substrates for three different intervals were obtained. Short deposition times $(27,45$ and $90 \mathrm{sec}$.) were used in order to preserve the (100) surface even for the smaller areas of growth. Images of the as-deposited surfaces were obtained by using an AFM in non-contact mode with a high-aspect ratio Si tip.

\section{Results and discussion}

Fig.1 shows the variation of surface morphologies viewed by the AFM for different growth times in both epi-ready and processed samples. For the former, surface features show up at early times (Fig.1a,b) and get elongated along a preferential direction as growth proceeds (Fig.1c). These features will lead eventually to the rippled structure observed at larger thicknesses by Cotta et al. [2]. For the processed samples, however, different height scales were observed for the InP morphologies. Fig.1d-f shows the surface imaged at large areas of

the processed sample. For early times, more pronounced, 3D, grain-like features are observed (Fig.1d). The peak-to-valley height variation is much larger than for the epi-ready substrates. These features also get larger and elongated as growth proceeds (Fig.1f), suggesting a coalescence process taking place.

Scans made inside the larger AFM patterns (Fig.2) reveal this same kind of structure at the center of the pattern. In the region close to the edges, however, a large growth rate enhancement is observed, forming structures where planes other than the (001) are present $[3,4]$. The enhanced nucleation at the edges competes with the nucleation at the center of the pattern. For patterns with smaller diversions, we observe a negligible thickness and no grain-like morphology in-between the structures at the edges.
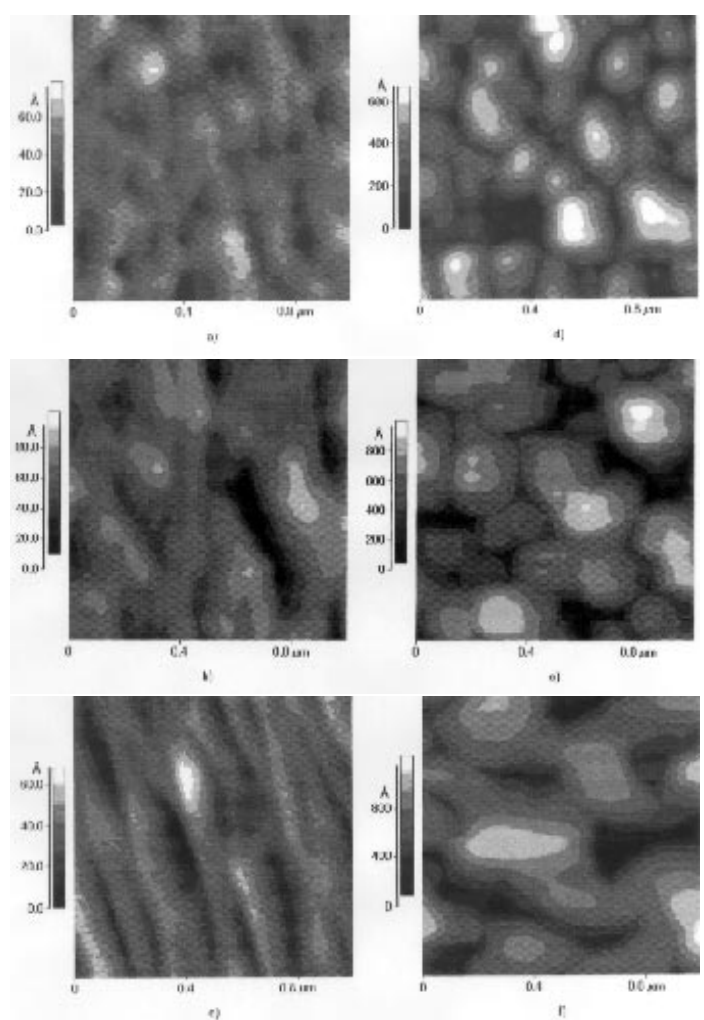

Figure 1. AFM images of the surface morphologies in InP/InP films grown over epi-ready substrates (during 27 $\sec (\mathrm{a}), 45 \sec (\mathrm{b}), 90 \sec (\mathrm{c}))$ and processed substrates (during $27 \sec (d), 45 \sec (e), 90 \sec (f))$, with a quasi $3 \mathrm{D}$-growth mode.
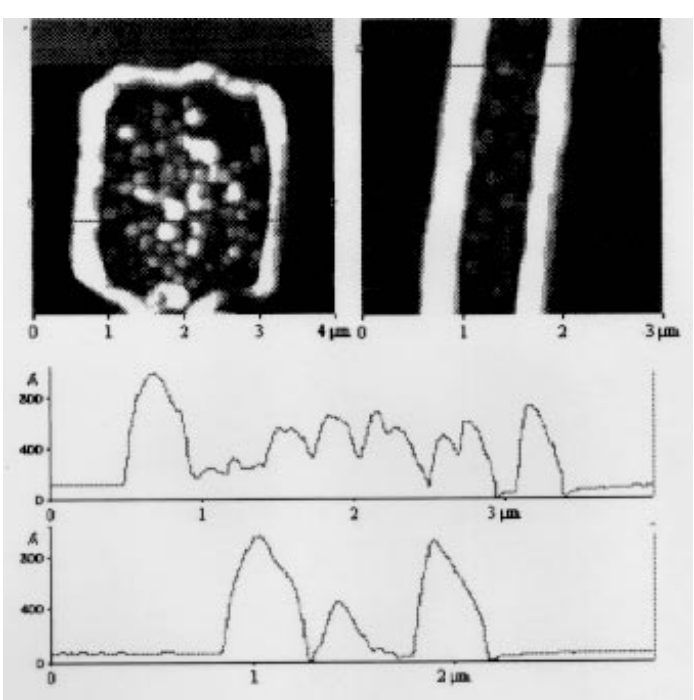

Figure 2. Images of the surface grown (in $3 \mathrm{~d}$-growth mode) on two patterns made by AFM lithography. The height profiles are measured along the straight lines over the surfaces.

Our results show that there are no significant differences between the AFM images of the AFM pattern centers and the large area of the processed samples. However, these morphologies, are very different from those observed for epi-ready surfaces. 
This behavior can be attributed to the processing of the surface for patterning. When the growth is carried out at conditions providing $2 \mathrm{D}$, layer-by-layer growth mode, on a processed substrate, a morphology similar to the epi-ready case is observed. Fig. 3 shows that a continuous film is obtained, with smooth morphologies and a larger density of oval defects than the film grown on the epi-ready substrate. These defects are usually attributed to surface contamination prior to growth [57]. It is also important to notice that the roughness of the processed samples alone can not be considered to cause the morphologies reported here. Roughness values for the processed surfaces are $\sim 5 \AA$ (peak to valley height variation $\sim 15 \AA$ ); similar values of roughness were obtained by long time annealing of epi-ready InP surfaces, with no significant effect on the morphologies of InP films grown subsequently on these A-surfaces [8]. In this sense residues left by the processing, in spite of the cleaning procedures used, are most likely at the origin of the different morphologies observed in this work for the epi-ready and processed samples.

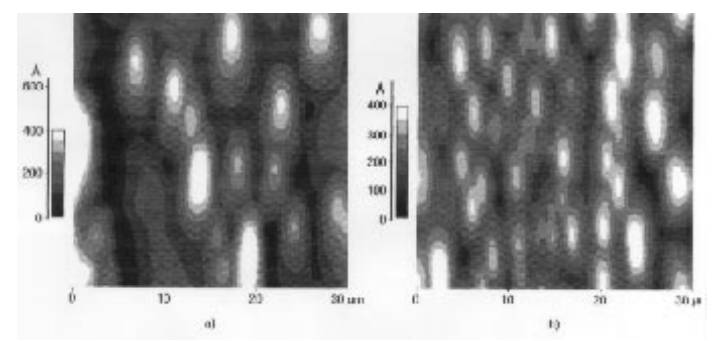

Figure 3. AFM images of the surface morphology for films grown on epi-ready (a) and processed (b) substrates with 2D-growth mode.

Since the growth was carried out using conditions providing a "quasi" 3D growth mode, diffusion is inhibited and the boundaries set by surface contamination should be more difficult to overcome. These boundaries could drive the growth to occur preferentially at particular regions of the surface (in the same sense as the edges of the pattern) with sizes in the sub-micron range (feature sizes $\sim 0.1-0.3 \mu \mathrm{m}$ ) and in most cases smaller than the dimensions of the AFM patterns.

In order to evaluate the existence of scaling properties for these surfaces, we have thus chosen to image small areas ( $\mathrm{L} \leq 0.2 \mu \mathrm{m}$ ) of the processed samples with the AFM. The scans were taken at the top region of the grain like features in three different regions of the sample: on the pattern edge (PE), on the grain-like structure at the center of the AFM pattern (GLP) and on the large areas in between - considered as an infinite surface (GLI). The film grown simultaneously on the epi-ready substrate (ER) was imaged by the AFM using similar scan sizes.
Fig.4 shows the temporal evolution of roughness for two different scan sizes L for both the processed (GLI) and epi-ready (ER) surfaces. We can see no well-defined growth exponent $\beta$ from these results. In fact, roughness unexpectedly decreases when growth time varies from 30 to $45 \mathrm{~s}$ for both samples. This behavior can correspond to the elongation of the surface features and consequent decrease of roughness along one direction. Also, for the shorter time, the influence of initial substrate surface roughness can not be totally neglected [9]. For longer times (90s), however, the roughness of the film on the epi-ready substrate remains approximately constant, while the processed sample shows a large increase in roughness value.

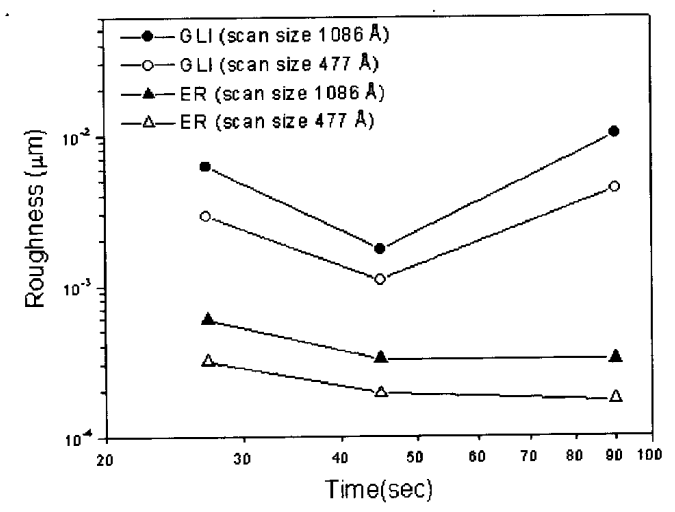

Figure 4. Temporal evolution of roughness for two different scan sizes L for both the processed (GLI) and epi-ready (ER) surfaces.

The typical picture for MBE growth, where roughness varies slowly with time [Tsao] agrees with the results for the epi-ready case. For the processed sample, the morphologies (Fig.1d-f) and roughness values (Fig.4) suggest cluster formation and growth, via coarsening and coalescence [10], due to the differences in the surface (in terms of surface contamination) where nucleation takes place. In order to investigate whether InP morphologies exhibit self-affine behavior, however, samples with longer growth times are required since the evolution of surface features and roughness could then be more clearly observed. On the other hand, when roughness and height-difference correlation functions are plotted against system size L, roughness exponents $\alpha$ can be measured. Fig. 5 and 6 show that processed samples provide $\alpha \geq 0.9$, for all three regions considered, while the epi-ready samples show $\alpha \simeq 0.75$. For the two-dimensional case, values of $\alpha$ closer to 1.0 are considered to describe film morphologies where surface diffusion is the main relaxation process [11,12], while smaller values of $\alpha$ (closer to $2 / 3$ ) indicate the existence of non-linear processes as well [1]. The lack of a defined $\beta$ value for these samples, however, prevent us from treating these surfaces as truly self-affine since a 
growth instability created, for example, by a diffusion bias on the surface [Stanley], could be present $[1,13]$. In this sense more experiments will be carried out in order to identify whether these surfaces exhibit self-affine properties and whether the change in the roughness exponents is related to changes in growth dynamics.

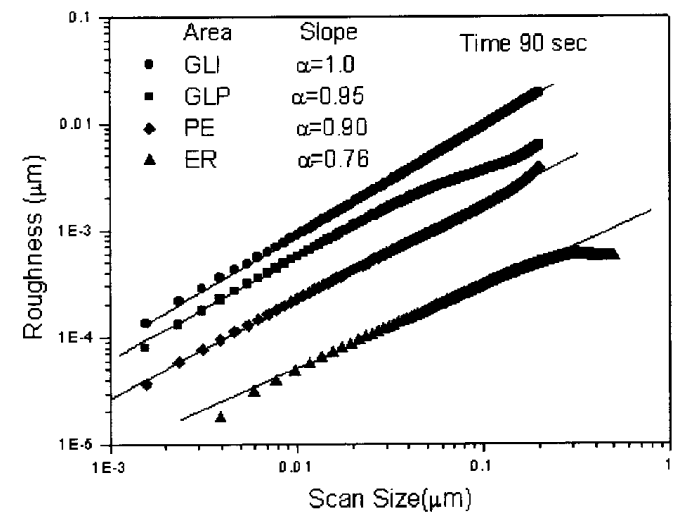

Figure 5. Scan size dependence of the surface roughness after a growth time of 90 sec., for different regions at the sample surface.

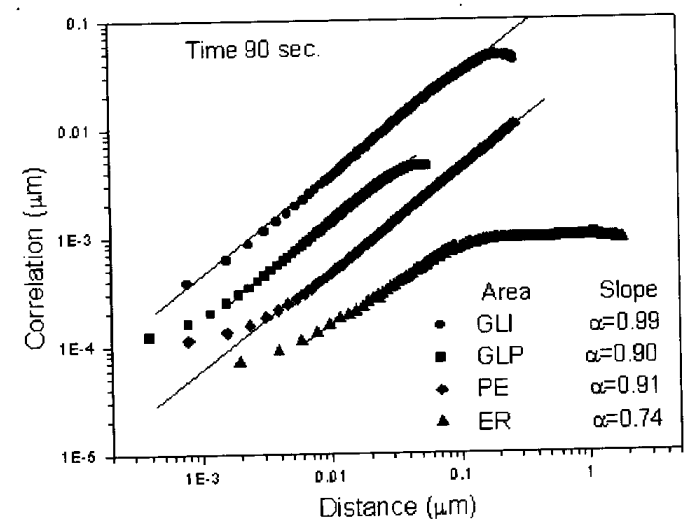

Figure 6. Height-difference correlation as function of the distance between surface sites, after a growth time of 90 sec.

In summary, we have observed changes in the morphology of InP films grown in epi-ready substrates and on patterns created by photo and plow-mode AFM lithography. These changes are attributed to different levels of contamination of the surface, which favor nucleation of clusters over a two-dimensional film for the lithography processed surface. The area of nucleation is smaller than the dimensions of the patterns created by lithography, so no size effects due to the presence of the pattern could be observed. The film morphologies exhibited well defined exponents for systems sizes smaller than $0.2 \mu \mathrm{m}$ but no defined growth exponent.

One of the authors (H.R.Gutiérrez) acknowledges financial support from $\mathrm{CNPq}$.

\section{References}

[1] Fractal Concepts in Surface Growth, A.L. Barabasi and H.E. Stanley, Cambridge University Press, Cambridge, 1995.

[2] M.A. Cotta, R.A. Hamm, T.W. Stanley, S.N.G. Chu, L.R. Harriott, M.B. Panish and H. Temkin, Phys. Rev. Lett. 70, 4106 (1993).

[3] H. Sugriera, T. Nishida, R. Iga, T. Yamada and T. Tamamura, J. Cryst. Growth 121, 579 (1992).

[4] F.S. Turco, M.C. Tamargo, D.M. Hwang, RE Nahory, J. Werner, K. Kash and E. Kapon, Apply. Phys. Lett. 56, 72 (1990).

[5] M.A. Cotta, R.A. Hamm, S.N.G. Chu, L.R. Harriott and H. Temkin, Apply. Phys. Lett. 66, 2358 (1995).

[6] C. Orme, M.D. Johnson, K.T. Leung and B.G. Orr, Mat. Sci. and Eng.B 30, 143 (1995).

[7] B.G. Orr, Workshop "Critical issues in Epitaxy" Boulder, USA, June 1994.

[8] L.S.M. Soares, M.A. Cotta, C.A.C. Mendonça, K.M.Ito-Landers and M.M.G de Carvalho, J. Cryst. Growth 191, 44 (1998).

[9] M.F. Gyure, J.J.Zinck, C.Ratsch and D.D. Vvedensky, Phys. Rev. Lett. 81, 4931 (1998).

[10] M. Zinhc- Allmang, L.C. Feldman and S. Nahahara, in Kinetics of ordering and growth at surface, ed. by M.G. Lagally, Plenum Press, NY, 1990, p. 455-472.

[11] D.E. Wolf and J. Villain, Europhys. Lett. B 13, 389 (1990).

[12] J. Villain, J. Phys. I 1, 19 (1991).

[13] P. Smilauer and D.D. Vvedensky, Phys. Rev. B 52, 14263 (1995). 